\title{
Discontinuous Shear Thickening in Cornstarch Suspensions
}

\author{
Abdoulaye Fall ${ }^{1, *}$, Anaël Lemaître ${ }^{1}$, and Guillaume Ovarlez ${ }^{2}$ \\ ${ }^{1}$ Laboratoire NAVIER, UMR 8205 CNRS-ENPC-IFSTTAR, 2 Allée Kepler, 77420 Champs sur Marne, France \\ ${ }^{2}$ CNRS, LOF, UMR 5258, 33600 Pessac, France
}

\begin{abstract}
We study the emergence of discontinuous shear-thickening (DST) in cornstarch, the well know system for this phenomenon, by combining macroscopic rheometry with local Magnetic Resonance Imaging (MRI) measurements. We bring evidence that macroscopic DST is characterized in wide gap with a shear localization, part of the material close to the inner cylinder is flowing and the rest is not. The flow seperates into a low-density flowing and a high-density jammed region. Moreover, the local rheology of the flowing region does not directly reflect DST but, strikingly, is most often shear-thinning. Our data are not consistent with recent theoretical suggestions based on the presumed existence of s-shaped flow curves. Instead, they support that DST should be attributed to the existence of a shear jamming limit at volume fractions quite significantly below random close packing.
\end{abstract}

\section{Introduction}

Granular suspensions are seemingly simple systems composed of non-colloidal rigid particles in Newtonian fluids, where the only relevant interactions are hydrodynamic and contact forces. Yet, they present a rich rheology [1], which includes shear-thinning or shear thickening, normal stress differences, shear banding and yield stress behaviour. With increasing packing fractions, continuous shear thickening (CST) appears at lower and lower strain rates and becomes more and more abrupt. This eventually leads to the emergence of a spectacular phenomenon: discontinuous shear thickening (DST), an order-of-magnitude jump of the stationary macroscopic stress when strain rate crosses some threshold value. DST is observed in a narrow range of packing fractions near random close packing. Recent works thus suggest that it is a dynamic or shear jamming transition, which motivates a considerable attention both from experiments [2], numerical simulation [3-5] and theory [6]. Shear thickening is also observed in dense colloidal suspension, where it has been related to the formation of dense clusters of particles [7-9]. In these systems, the competition between shear induced cluster formation and Brownian motion that homogenizes the suspension naturally determine a critical shear rate for the onset of shear thickening.

The origin of shear thickening is essentially unknown. Proposed mechanisms include: the crossover to Bagnold scaling due to particle inertia [10]; the coupling between normal and shear stresses associated with material dilatancy $[11,12]$. In numerical simulations DST was observed in systems which are forced to remain homogeneous by periodic boundary conditions [5]. It was thus suggested that it might arise from a jump of the local response, which occurs at fixed packing fraction, and is caused by the proliferation of frictional contacts $[5,6]$.

Examples abound, however, in granular suspension rheology where the macroscopic response is sharply different from the local behaviour: the apparent yield stress $[13,14]$ or a transient DST behavior [10], for example, were both shown to result from the emergence of flow inhomogeneities due respectively to density differences and migration. The observation of finite size effects in DST measurements [2,11], at sizes up to 100 particles, questions the idea that DST is a purely local phenomenon. In this context, it is unclear how a phase diagram for the local response can be deduced from experimental data and compared with numerical [5], and theoretical [6] works.

This situation motivates us to study DST in cornstarch [15], the well-known system for this phenomenon, while accessing experimentally the local response. To this aim, we have designed a new velocity controlled rheometer which can support torques up to $30 \mathrm{~N} . \mathrm{m}$ in a wide-gap Couette cell inserted in our Magnetic Resonance Imaging (MRI) scanner. It allows us to access local velocity and particle volume fraction profiles in the flowing sample [13], for a wide range of strain rates, which covers CST and DST. We thus bring evidence that macroscopic DST is characterized in wide gap with a shear localization, part of the material close to the inner cylinder is flowing and the rest is not. The flow separates into a low-density flowing and a high-density jammed region. Moreover, the local rheology of the flowing region does not directly reflect DST but, strikingly, is most often shear-thinning. Our data are not consistent with recent theoretical suggestions based on the

* Corresponding author: abdoulaye.fallifsttar.fr

C) The Authors, published by EDP Sciences. This is an open access article distributed under the terms of the Creative Commons Attribution License 4.0 (http://creativecommons.org/licenses/by/4.0/). 
presumed existence of s-shaped flow curves. Instead, they support that DST should be attributed to the existence of a shear jamming limit at volume fractions quite significantly below random close packing.

\section{Materials}

The cornstarch (Sigma) is the same as in [15]: it presents slightly polydisperse particles of size $\sim 20 \mu \mathrm{m}$ with irregular shapes (Fig. 1). We have measured the random close packing fraction $\phi_{R C P} \approx 55 \%$ by tapping the powder for long time in a container [16]. We have also measured the random loose packing $\phi_{R L P} \approx 35 \%$ as the density achieved after sedimentation of a dilute suspension [17]. The suspensions are prepared by mixing cornstarch in demineralized water. The initial volume fractions range from $33.5 \%$ to $43.9 \%$.

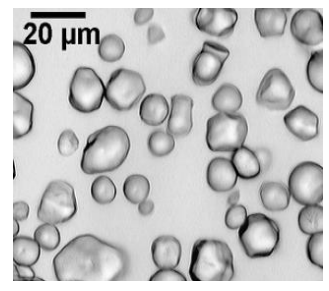

Fig.1: Micrograph of the cornstarch particles.

\section{Results}

Let us start with the macroscopic rheological behavior. Experiments are carried out with a Couette geometry on a Malvern Kinexus Pro stress-controlled rheometer. In order to avoid confinement effects, a wide gap is used [11]. The inner and outer cylinders are respectively of $25 \mathrm{~mm}$ and $36 \mathrm{~mm}$ diameter; both cylinders are roughened. The inner cylinder height is $37.5 \mathrm{~mm}$. Fig. 2a shows the evolution of torque measured on the inner cylinder during a 3 min logarithmic rotation rate $\Omega$ ramp from $5.10^{-3} \mathrm{rad} / \mathrm{s}$ to $200 \mathrm{rad} / \mathrm{s}$. near the inner cylinder, the local shear stress is $\tau\left(R_{i} ; \Omega\right)=T(\Omega) /\left(2 \pi R_{i}^{2} h\right)$ and the local strain rate is a priori unknown, but classically estimated as $\dot{\gamma}\left(R_{i} ; \Omega\right)=2 \Omega R_{o}^{2} /\left(R_{o}^{2}-R_{i}^{2}\right)$. Combining the stress and the strain rate equations yields the apparent viscosity $\eta \equiv \tau / \dot{\gamma}$ versus $\dot{\gamma}$ relation reported on Fig. $2 \mathrm{~b}$. Note that at low viscosities, macroscopic inertial effects may arise (Taylor Couette instabilities), which limit the accessible strain rate range at low volume fractions.

At low $\dot{\gamma}$, the response is clearly shear-thinning: the viscosity decreases with the shear rate with the exception of our highest packing fraction data, it crosses over to CST around a characteristic strain rate $\dot{\gamma}_{C S T}$ defined at that where the viscosity reaches a minimum. Viscosity jumps at some higher strain rate $\dot{\gamma}_{D S T}$ which characterizes the onset of DST. This behavior is generic: cornstarch suspensions from $38 \%$ to $44 \%$ volume fractions show a DST.
Since the classical estimate of $\dot{\gamma}\left(R_{i}\right)$, which is based on the Newtonian solution, remains unsatisfactory; an exact expression exists so long as the material is homogeneous in the studied torque range:

$$
\dot{\gamma}\left(R_{i} ; T_{o}\right)=\left.2 \sum_{n=0}^{\infty}\left(T \frac{d \Omega}{d T}\right)\right|_{T=T_{o}\left(\frac{R_{i}}{R_{o}}\right)^{2 n}}
$$

It cannot be used around the DST transition, however, because the apparent singularity of $d \Omega / d T$ cannot be resolved experimentally. Nevertheless we have checked that using the first two terms of (Eq. 1) gives the same qualitative behaviour as in Fig. 1b up to DST. We will show below that the flow does remain homogeneous below DST, which allows us to use (Eq. 1) to compute accurately $\dot{\gamma}_{C S T}$ and $\dot{\gamma}_{D S T}$ (Fig. 2c). Interestingly, (i) at any volume fraction, CST is always observed before DST; (ii) both $\dot{\gamma}_{C S T}$ and $\dot{\gamma}_{D S T}$ seem to vanish around the same volume fraction $\phi_{c}$ which we estimate to be $\approx 45 \%$ $\ll \phi_{R C P}$ by linear extrapolation.
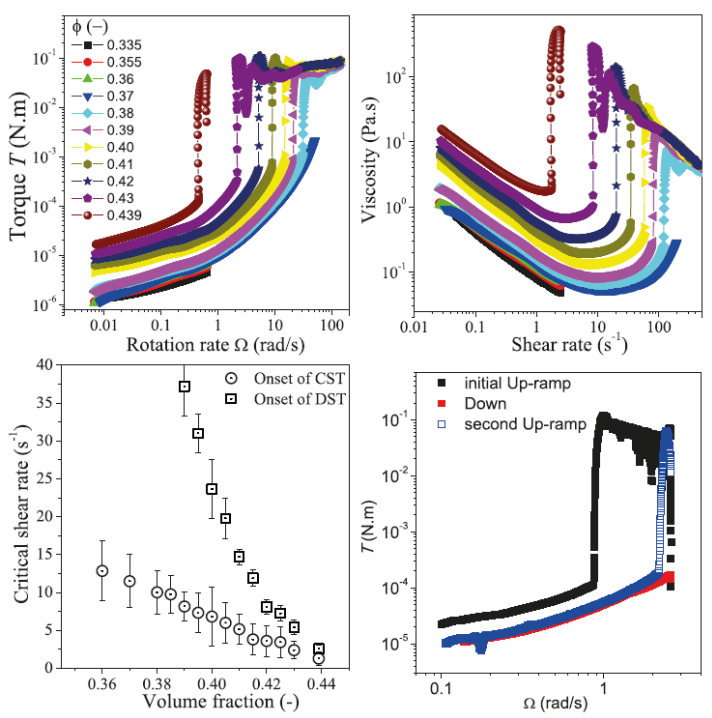

Fig.2: Macroscopic rheometry data. (a) Torque $T$ vs rotation rate $\Omega$ during a logarithmic ramp at various packing fractions $\phi$. (b) Apparent viscosity vs apparent shear rate extracted from (a). (c) Critical shear rates $\dot{\gamma}_{C S T}$ and $\dot{\gamma}_{D S T}$ vs $\phi$. (d) Reversibility test at $\phi=43.9 \%$ : succession of up, down, and up ramps.

To investigate further this thickening behavior, we look at the steady-state behavior when the shear-rate is increased up to a value $\dot{\gamma}>\dot{\gamma}_{D S T}$ and subsequently go down in rotation rate. We find that the system has completely changed: we descend on a completely different branch that is significantly below the original one. If we increase the shear (= rotation) rate again, we remain on this second branch; we can go up and down as long as we want but the system does not change anymore: there was an irreversible change in the system when we were on the first branch (Fig. 1d). 
In order to see what happens, we measure velocity and concentration profiles with the help of the velocitycontrolled MRI rheometry. Our Couette cell has inner and outer radii $R_{\mathrm{i}}=3 \mathrm{~cm}$ and $R_{\mathrm{o}}=5 \mathrm{~cm}$ (resp.) and inner cylinder height $h=11 \mathrm{~cm}$. Both cylinders are roughened to avoid slip, which we checked from velocity profiles. All experiments discussed below are performed by preparing a homogeneous material with mean volume fraction $\phi_{0}=43.9 \%$ (experiments at $40 \%, 41 \%$ and $42.5 \%$ show similar features), and imposing a fixed inner cylinder rotation rate $\Omega$ with values ranging between 5 and $100 \mathrm{rpm}$, until steady state is reached.

Our MRI $[13,18]$ provides the stationary packing fraction $\phi(r ; \Omega)$ and azimuthal velocity $v(r ; \Omega)$ at any radial position $r$. From the latter we extract the local strain rate $v(r ; \Omega)=v / r-\partial v / \partial r$. We do not have access to torque measurement. But, since the local stress is $\tau(r ; \Omega)=\tau\left(R_{i} ; \Omega\right) R_{i}^{2} / r^{2}$ in the Couette geometry, we can estimate the local viscosity profile as:

$$
\eta(r ; \Omega)=\frac{\tau\left(R_{i} ; \Omega\right)}{\dot{\gamma}(r ; \Omega)} \frac{R_{i}^{2}}{r^{2}}
$$

up to the unknow prefactor $\tau\left(R_{i} ; \Omega\right)$.

In Fig 3 , we plot the steady velocity and concentration profiles thus measured for a few $\Omega$ s. Velocity is normalized by its value at the inner cylinder.
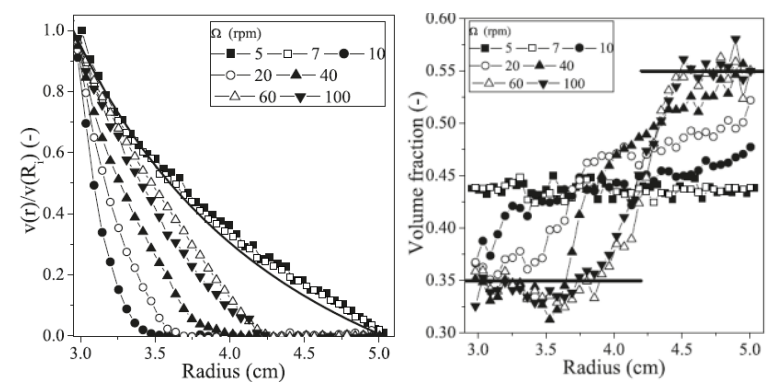

Fig.3: Steady MRI data for a $\phi_{0}=43.9 \%$ cornstarch suspension and different rotational velocities $\Omega$. (a) Velocity profiles. (b) Density profiles; solid lines indicate $\phi_{R L P}$ and $\phi_{R C P}$.

The results show that the system is well behaved and the particle concentration remains homogeneous when the imposed rotation rate is below a critical value around 10 rpm. However as soon as we go beyond this critical rotation rate, instead of decreasing continuously over the entire gap, the velocity profile goes to zero at some finite distance from the inner cylinder: there is shear localization. Upon increasing the rotation rate, we observe that the gap cannot be fully sheared: there still is a $8-25 \mathrm{~mm}$ region near the outer cylinder where the material is now not sheared, and for the highest rotation speeds, the sheared region does not occupy the whole gap of the Couette cell: the thickness of the sheared layer does not increase anymore whatever the imposed rotation rate and the concentration profile is essentially $\Omega$-independent in steady state.

This shear localization is associated with the sudden emergence of density inhomogeneities. Namely, the volume fraction decreases in the flowing layer, while it increases in the jammed region, as required by the conservation of particle number. Indeed, the concentration profile measurements (Fig. 3b) show that this happens because a strong gradient in particle concentration develops: the system shows particle migration. The result of the migration is that the particle concentration becomes smaller near the inner cylinder and larger near the outer cylinder. The latter observation also explains the velocity profile: a region develops that is so concentrated in particles that it is jammed. Interestingly to note that the concentration of this region corresponds to $\phi_{R C P}$. While the lower part of the concentration profile corresponds to $\phi_{R L P}$. It is noteworthy that the density profile can achieve multiple forms depending on shear history.

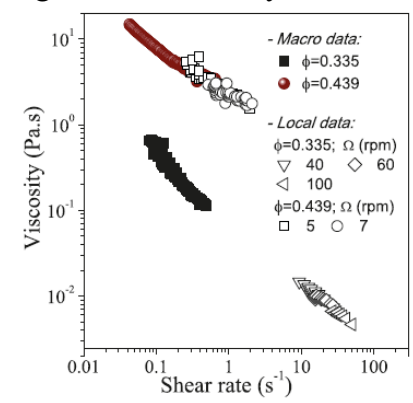

Fig.4: Comparison of local rheometry data obtained from MRI measurements (open symbols) and near the inner cylinder in macroscopic rheometry (filled symbols) in homogeneous conditions. Upper data: $\phi=43.9 \%$; lower data: $\phi \approx 33.5 \%$.

We now examine how the local rheology can be accessed within the shear-thickened flow. Three of our datasets, for $\Omega=40,60$, and 100rpm, present a finite width region of roughly uniform packing fraction $\phi_{\min } \simeq$ $33 \%$. Using only the data collected in these regions, we can hence implement the same approach as in the fully homogeneous case, to access the local rheology $\eta(\dot{\gamma} ; \phi)$ at $\phi=\phi_{\min }$. For each $\Omega$, we match the prefactor $\tau\left(R_{i} ; \Omega\right)$ and plot in Fig. 4 the resulting $\eta(\dot{\gamma} ; \phi)$ vs $\dot{\gamma}(r ; \Omega)$ data points. Clearly, the local $\eta\left(\dot{\gamma} ; \phi_{\min }\right)$ rheology is shearthinning, and extrapolates the $\phi=33.5 \%$ rheometry measurements. These data demonstrate that macroscopic DST cannot be interpreted as a reflecting DST in the local response at a fixed volume fraction. Instead, it results from the separation of the system into a low density flowing and a high density jammed regions. Somewhat paradoxically, the local response was found above to be shear-thinning in the flowing layer of the macroscopic shear-thickened state. In the flowing layer, the density plateau only develops around $\phi_{\min }$. It is hence at this packing fraction only that we can extract the local rheology $\eta(\dot{\gamma} ; \phi)$ from MRI data. To further qualify the local response, we now construct a phase diagram for all the local flow and jammed states. To this end, we collect the operating points $(\dot{\gamma}(r ; \Omega), \phi(r ; \Omega))$ obtained with the MRI for all $\Omega$ 's and all $r$ 's and report them on Fig. 5, where they are compared with the curves $\dot{\gamma}_{C S T}(\phi)$ and $\dot{\gamma}_{D S T}(\phi)$ that delimit the flow regimes previously 
identified using macroscopic rheometry. Our MRI data include points obtained in the flowing layer as well as in jammed regions: in the scatter plot, they are respectively found on the left (low $\phi$, with non-vanishing $\dot{\gamma}$ ) and right (high $\phi, \dot{\gamma} \simeq 0$ ) hand sides.

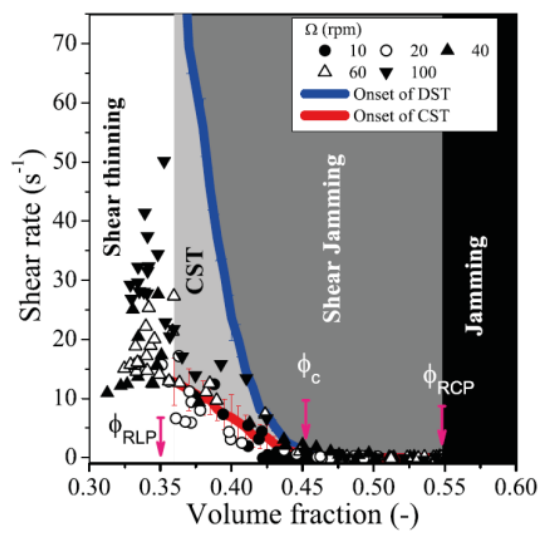

Fig.5: Phase diagram: scatter plot of the local $(\dot{\gamma}(r ; \Omega), \phi(r ; \Omega))$ values compared with the $\dot{\gamma}_{C S T}(\phi)$ and $\dot{\gamma}_{D S T}(\phi)$ lines.

All the local flow points clearly lie below the $\dot{\gamma}_{D S T}(\phi)$ line, which thus appears to be an intrinsic material limit beyond which no steady flow can be sustained. No flow is found in particular at packing fractions above the value $\phi_{c} \simeq 45 \%$ where $\dot{\gamma}_{C S T}(\phi)$ and $\dot{\gamma}_{D S T}(\phi)$ vanish. $\phi_{c} \ll$ $\phi_{R C P} \simeq 55 \%$ is quite distinct from the jamming point, and a continuum of jammed states is thus accessed beween $\phi_{c}$ and $\phi_{R C P}$.

\section{Conclusion}

In the literature, macroscopic DST was proposed to result from the coexistence, in a range of packing fractions, of two flow curves for the local response. This bi-stability is justified by introducing an auxiliary state variable, such as the density of frictional contacts. Within this viewpoint, at low strain rate, the local response lies on a low-viscosity curve; DST occurs when the flow becomes unstable and jumps on high-viscosity curve. Macroscopic DST would thus result from an intrinsic DST behavior of the local response. Our data, however, supports a completely different scenario. Indeed, at the onset of macroscopic DST, the part of the flow that reaches locally a limit of stability, i.e. the $\dot{\gamma}_{D S T}$ line, is the region near the inner cylinder, where the local shear rate is maximal. Within existing interpretations of DST based on s-shaped flow curves, it is this locally unstable region that should jump to a high viscosity state. But, in stark contrast, we find that when DST occurs, this region jumps toward a low-density, low viscosity shear-thinning state. Meanwhile jamming occurs in the region near the outer cylinder which, at the onset of DST, was well within its stability limit. DST thus occurs when a global reorganization of the flow takes place. It does not naively reflect local jumps of the local rheology.
Hence we do not find any necessity to account for a secondary flow curve associated with a change of a state variable such as a density of contacts. Instead, DST is clearly determined by two major factors: (i) the existence of a limit $\dot{\gamma}_{D S T}(\phi)$ beyond which no steady flow can be accessed; (ii) the fact that as $\phi_{c} \ll \phi_{R C P}$, a continuum of jammed states exists. Both are direct consequences of a unique physical phenomenon: shear jamming. We thus understand that when the inner cylinder region reaches the shear-jamming limit, it can only escape the $\dot{\gamma}_{D S T}(\phi)$ line by decreasing its local volume fraction, thus driving migration throughout the gap. This is possible because density can be increased significantly above $\phi_{c}$ in the growing jammed layer. Many questions, of course, remain especially about the microscopic mechanisms which lead to the emergence of a shear-jamming line at packing fraction quite separated from $\phi_{R C P}$. It is quite plausible that the angularity of particles, which is significant for cornstarch, is a determining factor.

\section{References}

[1] M. M. Denn and J. F. Morris, Annu. Rev. Chem. Biomol. Eng. 5, 203 (2014).

[2] E. Brown, H. Zhang, N. A. Forman, B. W. Maynor, D. E. Betts, J. M. DeSimone, and H. M. Jaeger, J. Rheol. (N. Y. N. Y). 54, 1023 (2010).

[3] R. Seto, R. Mari, J. F. Morris, and M. M. Denn, Phys. Rev. Lett. 111, (2013).

[4] C. Heussinger, Phys. Rev. E - Stat. Nonlinear, Soft Matter Phys. 88, (2013).

[5] R. Mari, R. Seto, J. F. Morris, and M. M. Denn, J. Rheol. (N. Y. N. Y). 58, (2014).

[6] M. Wyart and M. E. Cates, Phys. Rev. Lett. 112, 1 (2014).

[7] J. Bender, J. Rheol. (N. Y. N. Y). 40, 899 (1996).

[8] B. J. Maranzano and N. J. Wagner, J. Chem. Phys. 114, 10514 (2001).

[9] N. J. Wagner and J. F. Brady, Phys. Today 62, 27 (2009).

[10] A. Fall, A. Lemaître, F. Bertrand, D. Bonn, and G. Ovarlez, Phys. Rev. Lett. 105, (2010).

[11] A. Fall, N. Huang, F. Bertrand, G. Ovarlez, and D. Bonn, Phys. Rev. Lett. 100, (2008).

[12] A. Fall, F. Bertrand, G. Ovarlez, and D. Bonn, J. Rheol. (N. Y. N. Y). 56, (2012).

[13] G. Ovarlez, F. Bertrand, and S. Rodts, J. Rheol. (N. Y. N. Y). 50, 1 (2006).

[14] A. Fall, F. Bertrand, G. Ovarlez, and D. Bonn, Phys. Rev. Lett. 103, (2009).

[15] F. S. Merkt, R. D. Deegan, D. I. Goldman, E. C. Rericha, and H. L. Swinney, Phys. Rev. Lett. 92, 184501 (2004).

[16] P. Philippe and D. Bideau, Europhys. Lett. 60, 677 (2002).

[17] M. P. Ciamarra and A. Coniglio, Phys. Rev. Lett. 101, (2008).

[18] A. Fall, F. Bertrand, D. Hautemayou, C. Mezière, P. Moucheront, A. Lemaître, and G. Ovarlez, Phys. Rev. Lett. 114, 1 (2015). 